\title{
ON THE STABILITY OF INVERTER-BASED MICROGRIDS VIA LMI OPTIMIZATION
}

\author{
TRUONG DUC TRUNG AND MIGUEL PARADA CONTZEN \\ Control systems group, Technical University Berlin \\ \{trung,parada-contzen\}@control.tu-berlin.de
}

\begin{abstract}
The implementation of a number of individual small distributed energy resources forms the new generation of power systems - the microgrid. For an isolated operating condition of microgrids, the classical stabilizing approaches for control of large power systems are no longer applicable, as the characteristics of microgrids differ significantly from conventional power systems. Therefore, a new control approach must be investigated in order to robustly stabilize microgrids during disturbances, which are caused by load changes and the intermittent nature of alternative energy sources. In the present paper, the stability of inverter-based microgrids is considered. A decentralized state-feedback control approach for inverter-based microgrids with a linear matrix inequality (LMI) stability condition is proposed. Controller gains for inverters are designed by solving the LMI optimization problem. The resulting controller stabilizes the system, guaranteeing zero steady-state frequency deviations. The control approach is then validated via an academical example.

Keywords. decentralized control, distributed energy resource, LMI, microgrids, quasi-block diagonal dominance, voltage-source inverters.
\end{abstract}

\section{INTRODUCTION}

The widespread implementation of renewable energy sources, e.g. wind, solar energy, biogas, etc., leads to an increasing amount of distributed energy resources (DERs), i.e. windturbines, photovoltaics, fuel cells, etc., which are connected to the transmission network at the low-voltage (LV) level next to the consumption point. Most of DERs are natively direct current (DC) or unregulated alternative current $(\mathrm{AC})$ resources, while conventional power networks are normalized AC systems. Thus, DERs are interfaced to the transmission network through power electronic devices called inverters. In order to take control over those small generation units, the concept of microgrids was introduced [1,2]. A microgrid characterized by a combination of DERs with inverter interfaces is called an inverter-based microgrid and its generation units are voltage source inverters. A microgrid can operate in connected mode with a transmission network and execute power exchange with it. In this case, the transmission network is dominant and the microgrid is considered as a single load or a single generation unit. Moreover, a microgrid can separate itself and start operating in isolated mode when there are detected faults in the transmission network [1].

As reported in a number of publications, there appear many technical problems associated to stability and performance in the sense of voltage and frequency in inverter-based microgrids while operating in isolated mode $[1,3,4]$. Usually, most of generation units in microgrids have low inertias due to their small sizes and capacities. Besides, due to the intermittent nature of renewable energy sources, i.e. inconsistent sunshine or wind, some generation units are possibly unavailable during operation. Therefore, it is a control challenge to maintain operation of microgrids with acceptable 
deviations around the nominal values of voltages and frequencies in the presence of disturbances from load uncertainties and natural sources.

A microgrid can contain a large number of generation units, which must be controlled by decentralized controllers for the system to be largely independent on the communication [1]. Therefore, the decentralized stabilizing controllers of inverters based on the local measurement must guarantee the stability of the system on a global scale. Decentralized controllers must allow inverters to work in a plug-and-play and peer-to-peer manner, which means that all inverters are equally treated and each inverter can smoothly connect and disconnect from the system without harming its stability and operation $[1,3,4]$. Moreover, another important issue associated to the operation of a microgrid is the power sharing, that is, all the generation units in the system should share any increase in load by predefined power sharing ratios. This control task should be performed as well by inverter decentralized controllers.

An important decentralized control approach applied to microgrids is the droop control ${ }^{1}$, which is widely implemented in large power systems with synchronous generators (SGs). However, as reported in literature $[4,6,7,8]$, the droop control experiences many drawbacks when it is applied to inverterbased microgrids. The main reason is that the characteristics of microgrids differ significantly from the characteristics of large power systems. For instance, microgrids operate at LV levels, where ratios between connecting line resistances and reactances (known as $R / X$ ratios) are considerable, making the assumption of the droop control on purely inductive connecting lines between generation units no longer accurate. Moreover, while working with inverters, i.e. power electronic devices, there is no inherent physical relation between network frequency and power balance as in power systems with SGs. Consequently, the droop control results in poor transient performance as well as lack of robustness in inverter-based microgrids $[6,7]$.

As the connecting lines between generation units in LV microgrids are no longer purely inductive, a coupling between the $f / P$ and $V / Q$ droop control loops exists, which is reported as the main drawback of the droop control to stabilize microgrids and achieve desired power sharing $[9,10,4]$. Regarding this issue, line resistances are included in the droop-based control approaches proposed in $[?, 4,11,8,12]$. Alternative droop control methods $[4, ?]$ have been developed in order to include the couplings between $f / P$ and $V / Q$ in the controller design by adding $V / P$ and $f / Q$ control loops to the classical droop control. The active and reactive power are then modified by both frequency and voltage droops. A technique called virtual output impedance was proposed in $[13,8,11]$ to reduce virtually the ratio $R / X$ of connecting lines by adding a new output control loop, thereby improving the stability margin and power sharing ratio in microgrids. These methods perform better power sharing and reduce partially the coupling between active and reactive power control loops in particular cases of microgrids. However, no analytical solution is given to guarantee the stability of inverter-based microgrids.

Regarding the stability drawback of the droop-based control approaches, several alternative control strategies were proposed. In $[14,15,16]$ the instability of microgrids was reported due to the low-inertia nature of DERs, in contrast to SGs with rotating masses. Dealing with this matter, the concepts of virtual SG and synchronverter to combine inverter technology with properties of SGs were introduced. Thus far, those techniques are still not mature and need to be further studied. Other alternatives, such as the master/slave approach proposed in [17] or the communication-based control method presented in [18], can improve the stability margin of microgrids. However, these methods do

\footnotetext{
${ }^{1}$ The droop control consists of two control loops denoted by $f / P$ and $V / Q$ that by adjusting active and reactive power independently, frequency and voltage magnitude are determined, respectively [5].
} 
not satisfy the desirable communicationless decentralized control, and therefore, may be not realistic for microgrid applications.

The above discussion has just briefly reviewed some research activities in the microgrid control field. To the best of our knowledge, neither the droop-based nor the alternative control approaches can actually give analytical solutions to guarantee the stability and performance of microgrids. Hence, those control issues are still an open research field.

As the stability of inverter-based microgrids is claimed to be difficult to achieve, consequently a thorough stability analysis needs to be investigated, which is the main object of the present paper. It is worth mentioning that we separate the stability and the power sharing issues, considering power sharing as a performance criterion of inverters, which can be included only when the system stability is guaranteed. Some comments on the power sharing are also given in the paper. However, a detailed discussion on this topic can be found in our previous works $[6,7]$.

The main contributions of the paper are twofold. First, a model of inverter-based microgrids and a decentralized control approach with an LMI stability condition are proposed. It will be shown that the control approach guarantees the system stability and zero steady-state frequency deviations. Opposed to the droop-based controls, the authors do not intend to decouple the power control loops of a single inverter, but rather implement all possible local measurements to assure the system stability. Thus, output power of inverters are modified by drooping phase angles and voltage magnitudes. Second, as microgrids are highly coupled systems [4], resulting in low stability margin and poor performance, the LMI stability condition is extended to target a quasi-block diagonal dominant closed-loop microgrid. This results in reduced influences of the interconnection between inverters on the overall system stability and performance. Consequently, the stability margin of the system is increased.

The paper is organized as follows. Section 2 introduces a model of an inverter-based microgrid. In section 3, a stabilizing decentralized control approach for inverter-based microgrids is proposed. Then, an LMI stability condition is presented to complete the control approach. Section 4 discusses the quasi-block diagonal dominance of closed-loop microgrids. An academical example is given in section 5 to support the proposed control approach. Finally, conclusions and future research directions are given in section 6 .

\section{MODELING OF INVERTER-BASED MICROGRIDS}

In a microgrid, inverters and loads are connected to each other in an arbitrary manner. It is assumed constant impedance loads, then the system can be presented equivalently by using the standard Kron reduction technique to eliminate passive nodes [19]. Each inverter represents one active node of the reduced network. A Kron-reduced structure of an inverter-based microgrid with $n$ inverters is shown in Fig. 1, which is the considered case throughout the paper.

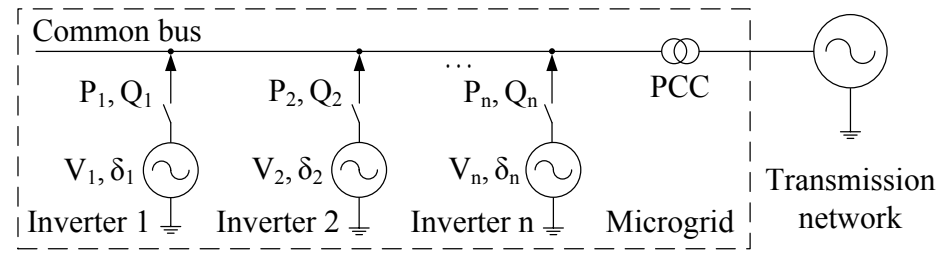

Figure 1: Schematic representation of an inverter-based microgrid.

It is well known that the control system for inverters obtains a three-level structure [5]. The control of the inverter flux vector forms the innermost control level, which controls directly the 
inverter switching. The middle level controls the frequency and magnitude of the inverter output voltage, providing set points for the innermost control loop. The set points for the middle control level are obtained from the outermost loop - the power control loop. The switching frequency of inverters is in the range about $8-20[\mathrm{kHz}]$, which is much faster than rated frequencies of power systems, e.g., $50[\mathrm{~Hz}]$. Moreover, the inverter output power is required to drive the power control loop proposed later. The output power is measured through a low-pass filter, which makes the bandwidth of the power control loop much smaller than the bandwidth of the voltage control loop. Hence, dynamics of the innermost and the middle control loops are much faster than dynamics of the power control loop, which is strongly influenced by the low-pass filter.

Based on the facts above, for the stability analysis of inverter-based microgrids the following assumptions are made. An ideal voltage source on the DC-side of each inverter is assumed. All inverters are equally treated as voltage sources inverters with controllable output voltages $V_{i}$ and phase angles $\delta_{i}$. Moreover, the case of $i d e a l$ voltage source inverters is assumed, i.e. only the power control loop of inverters is explicitly considered, while dynamics of lower control levels are assumed to be exceedingly fast and can be neglected. This is a relatively safe assumption for stability analysis of microgrids at the power control level, which causes most stability problems [20]. The lower level control loops are assumed to perfectly and rapidly track their references [3].

Based on the above assumptions of the considered microgrid, the active power $P_{i}$ and reactive power $Q_{i}$ exchanged at each node $i$ of the system are expressed by the following standard power flow equations [19]

$$
P_{i}=\sum_{j=1}^{n} V_{i} V_{j}\left|Y_{i j}\right| \cos \left(\delta_{i}-\delta_{j}-\phi_{i j}\right), \quad Q_{i}=\sum_{j=1}^{n} V_{i} V_{j}\left|Y_{i j}\right| \sin \left(\delta_{i}-\delta_{j}-\phi_{i j}\right),
$$

where $\delta_{i}, \delta_{j}$ are phase angles, $V_{i}, V_{j}$ voltage magnitudes, $\left|Y_{i j}\right|$ and $\phi_{i j}$ the absolute value and the angle of an admittance $Y_{i j}$ between node $i$ and node $j$.

All phase angles are expressed with respect to a common rotating reference frame with a stationary angular velocity $\omega_{\text {nom }}$, which is equal to the system rated frequency. The active and reactive power are then measured through a low-pass filter as follows

$$
\tilde{P}_{i}=\frac{P_{i}}{\tau_{i} s+1}, \quad \tilde{Q}_{i}=\frac{Q_{i}}{\tau_{i} s+1}
$$

where $\tilde{P}_{i}$ and $\tilde{Q}_{i}$ are the measured active and reactive power, $\tau_{i}$ is the time constant of the filter, and $s$ is the Laplace variable.

In order to investigate the stability of a microgrid around an equilibrium point, the state-space model of the system with the state variable $x(t)$ and control input $u(t)$ are defined as follows

$$
\begin{aligned}
x_{i} & =\left[\delta_{i}-\delta_{i_{0}}, \tilde{P}_{i}-\tilde{P}_{i_{0}}, \tilde{Q}_{i}-\tilde{Q}_{i_{0}}\right]^{T}, & u_{i} & =\left[\dot{\delta}_{i}, V_{i}-V_{i_{0}}\right]^{T}, \\
x & =\left[x_{1}^{T}, \ldots, x_{n}^{T}\right]^{T}, & u & =\left[u_{1}^{T}, \ldots, u_{n}^{T}\right]^{T},
\end{aligned}
$$

where the nominal equilibrium point of each inverter $i$ is

$$
x_{i_{0}}=\left[\delta_{i_{0}}, \tilde{P}_{i_{0}}, \tilde{Q}_{i_{0}}\right]^{T}, \quad u_{i}=\left[0, V_{i_{0}}\right]^{T} .
$$

As seen in (1) that $P_{i}, Q_{i}$ can be modified by varying the phase angles and the voltage magnitudes. However, by taking the idea of the droop control, the frequency $\dot{\delta}_{i}$ is controlled instead of direct 
modification of the phase angle $\delta_{i}$. Moreover, it will be shown that inverter frequencies always converge to a common rated value. Thus, the selected system variables refer to an angle droop control and a voltage droop control.

Remark 1: The system stability with respect to the variables (3) and the nominal equilibrium point (4) indicates the nominal system stability. However, the equilibrium point of a power system is often not completely known beforehand and changes during operation, depending on the system topology and load conditions. This results in new equilibrium points, and invalidates the variables (3). Regarding this matter, along with a linear time-invariant (LTI) system model, load uncertainties will be considered in our future work. The authors will also extend the controller design in oder to guarantee robustly the system stability despite load uncertainties. In this paper, a linear system model of a nonlinear microgrid is investigated, assuming a level of robustness of the microgrid around the interested equilibrium point (4).

The state-space model of an inverter $i$ is presented by the following ordinary differential equations

$$
\left\{\begin{array}{l}
\dot{\delta}_{i}=\omega_{i} \\
\dot{\tilde{P}}_{i}=\frac{-\tilde{P}_{i}+P_{i}}{\tau_{i}} \\
\dot{\tilde{Q}}_{i}=\frac{-\tilde{Q}_{i}+Q_{i}}{\tau_{i}}
\end{array}\right.
$$

where $\omega_{i}$ is the inverter output frequency, $P_{i}$ and $Q_{i}$ are given in (1). Then, from linearizing equations (1) around the interested operating point (4), an LTI state-space model of the system derives as

$$
\left\{\begin{array}{l}
\dot{x}(t)=A x(t)+B u(t) \\
y(t)=C x(t)=x(t)
\end{array}\right.
$$

and each inverter $i$ is related to one subsystem with the following state-space model

$$
\dot{x}_{i}(t)=A_{i i} x_{i}(t)+B_{i i} u_{i}(t)+\sum_{j=1}^{n-1}\left(A_{i j} x_{j}(t)+B_{i j} u_{j}(t)\right),
$$

where $A \in \mathbb{R}^{3 n \times 3 n}, B \in \mathbb{R}^{3 n \times 2 n}, C=I_{3 n \times 3 n}$ and

$$
A_{i i}=\left[\begin{array}{ccc}
0 & 0 & 0 \\
\frac{\partial P_{i}}{\tau_{i} \partial \delta_{i}} & \frac{-1}{\tau_{i}} & 0 \\
\frac{\partial Q_{i}}{\tau_{i} \partial \delta_{i}} & 0 & \frac{-1}{\tau_{i}}
\end{array}\right], B_{i i}=\left[\begin{array}{cc}
1 & 0 \\
0 & \frac{\partial P_{i}}{\tau_{i} \partial V_{i}} \\
0 & \frac{\partial Q_{i}}{\tau_{i} \partial V_{i}}
\end{array}\right], A_{i j}=\left[\begin{array}{ccc}
0 & 0 & 0 \\
\frac{\partial P_{i}}{\tau_{i} \partial \delta_{j}} & 0 & 0 \\
\frac{\partial Q_{i}}{\tau_{i} \partial \delta_{j}} & 0 & 0
\end{array}\right], B_{i j}=\left[\begin{array}{cc}
0 & 0 \\
0 & \frac{\partial P_{i}}{\tau_{i} \partial V_{j}} \\
0 & \frac{\partial Q_{i}}{\tau_{i} \partial V_{j}}
\end{array}\right]
$$

Inverters are interconnected through their state variables and control inputs, which are specified by the matrices $A_{i j}$ and $B_{i j}$. Whereas, $A_{i i}$ and $B_{i i}$ are system matrices of each inverter.

Remark 2: Due to the fact that matrix $A$ possesses zero eigenvalues, the matrix $[A-\lambda I, B]$ does not have full-row rank with all $\lambda \in \mathbb{C}$, where $\lambda$ is the eigenvalue of $A$. The system (6) is therefore not controllable. However, $[A-\lambda I, B]$ has full-row rank for all $\lambda$ with $\operatorname{Re}(\lambda) \geq 0$. Hence, the system is stabilizable, and a state-feedback controller $K$ exists, so that the system is stable (i.e. $A+B K$ is stable) [21].

Problem 1: Design local state-feedback controllers $K_{i}: u_{i}(t)=K_{i} x_{i}(t), i=1, \ldots, n$ for each subsystem (7) to stabilize the overall interconnected system (6), and the controller of the overall 
system is restricted to be block-diagonal as follows

$$
u(t)=K x(t), \quad K=\operatorname{diag}\left(K_{1}, \ldots, K_{n}\right) .
$$

In order to analyze the quasi-block diagonal dominance of the closed-loop system in section 4 , the diagonal $A_{d}, B_{d}$ and off-diagonal parts $A_{H}, B_{H}$ of the matrices $A, B$ are defined as: $A_{d}=$ $\operatorname{diag}\left(A_{11}, \ldots, A_{n n}\right), A_{H}=A-A_{d}, B_{d}=\operatorname{diag}\left(B_{11}, \ldots, B_{n n}\right), B_{H}=B-B_{d}$. Define $A_{\mathrm{cl}}:=$ $A_{d}+B_{d} K$, then the state-space model of the closed-loop system with the controller $K$ can be written as the following

$$
\left\{\begin{array}{l}
\dot{x}(t)=A_{\mathrm{cl}} x(t)+\left(A_{H}+B_{H} K\right) x(t) \\
y(t)=x(t)
\end{array}\right.
$$

\section{STATE-FEEDBACK STABILIZING CONTROLLER DESIGN}

In this section, the authors propose a decentralized controller design based on the LMI technique, which guarantees stability of the system (9). The control loop of a single inverter is shown in Fig. 2. The output current $I_{i}$, voltage $V_{i}$, and the phase angle $\delta_{i}$ are measured in order to calculate the active and reactive power. The phase angles of all inverters are measured with respect to a common rotating reference frame with an angular velocity $\omega_{\text {nom }}$, which is created by the clock synchronization. Therefore, the GPS signal ${ }^{2}$ is required to synchronize the clocks, but no other communication link between inverters is needed [23, 24, 22].

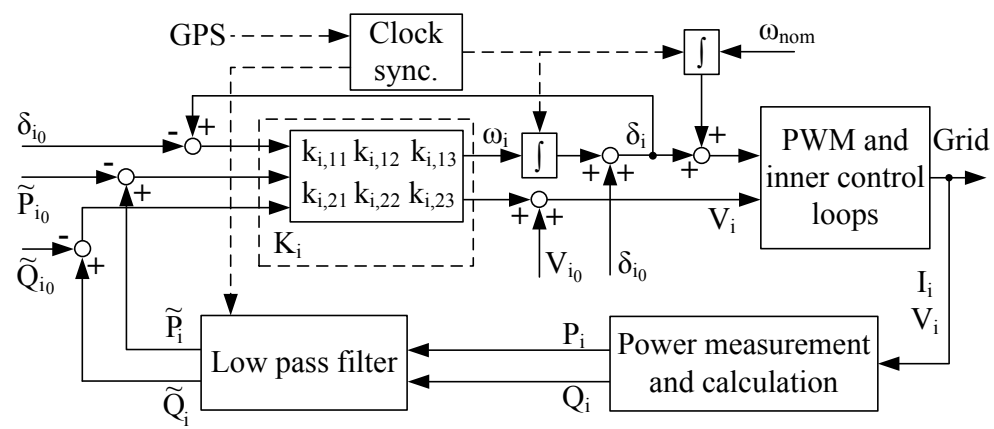

Figure 2: Power control loop of an inverter.

In the proposed system model, the deviation of the phase angle from its reference $\left(\delta_{i}-\delta_{i_{0}}\right)$ is defined as a state of an inverter $i$. Hence, if the controller $K$ stabilizes the microgrid, the variations of $\delta_{i}(t), i=1, \ldots, n$ will be stabilized so that $\Delta \delta_{i}=$ const., which infers the zero steady-state deviation of the inverter output frequency as $\Delta \omega_{i}=\dot{\delta}_{i}=0$. The presented control approach has an advantage over the droop control since it does not need any additional secondary control for frequency restoration. It is consistent with the nature of microgrids with power electronic interfaces, where there is no inherent relation between network frequency and the power balance in the system. Instead, according to the power flow equations (1) the power balance is obtained by drooping phase angles and magnitudes of voltages with respect to their nominal values.

Regarding the power sharing issue, it will be validated via simulation that in order to ensure stability of the system, all the inverters must generate power to supply the load demand. As a consequence, there is power exchange between system nodes, namely, the power sharing. As discussed

\footnotetext{
${ }^{2}$ In the case the GPS input of an inverter is lost for several seconds, the local clock can be implemented to create a sufficient rotating reference frame for the inverter [22].
} 
in our previous works $[6,7]$, by adjusting the controller gains without causing system instability, more power exchanges can be achieved. However, no claim on power sharing performance could be made. To the best of our knowledge, none of the proposed control methods gives an analytical solution to ensure a desired power sharing in microgrids, especially in the case of reactive power sharing.

Theorem 1. Consider the closed-loop system (9). Let $P \in \mathbb{R}^{3 n \times 3 n}$ be a symmetric positive definite matrix and $P=\operatorname{diag}\left(P_{11}, \ldots, P_{n n}\right)$, where $n$ is the number of inverters in the system. Define

$$
\mathbf{X}=\operatorname{diag}\left(\mathbf{X}_{11}, \ldots, \mathbf{X}_{n n}\right)=P^{-1}, \quad \mathbf{Y}=\operatorname{diag}\left(\mathbf{Y}_{11}, \ldots, \mathbf{Y}_{n n}\right)=K P^{-1},
$$

then the block-diagonal controller $K=\mathbf{Y} \mathbf{X}^{-1} \in \mathbb{R}^{2 n \times 3 n}$ guarantees the stability of the system (9), if the following LMI is feasible

$$
\left[\begin{array}{ccccc}
A_{d} \mathbf{X}+B_{d} \mathbf{Y}+\left(A_{d} \mathbf{X}+B_{d} \mathbf{Y}\right)^{T} & A_{H} & B_{H} & \mathbf{X} & \mathbf{Y}^{T} \\
A_{H}^{T} & -I & 0 & 0 & 0 \\
B_{H}^{T} & 0 & -I & 0 & 0 \\
\mathbf{X} & 0 & 0 & -I & 0 \\
\mathbf{Y} & 0 & 0 & 0 & -I
\end{array}\right] \leq 0 .
$$

Proof. The proof of theorem 1 uses Lyapunov's stability theorem and is inspired by [25]. Let the selected Lyapunov function associated to the closed-loop system (9) be given in the form $\mathcal{V}(x)=$ $x^{T} P x$, with a symmetric positive definite matrix $P$. Then, with (9), the derivative of the Lyapunov function $\mathcal{V}(x)$ is obtained as follows

$$
\dot{\mathcal{V}}(x)=\dot{x}^{T} P x+x^{T} P \dot{x}=x^{T}\left(A_{\mathrm{cl}}^{T} P+P A_{\mathrm{cl}}+A_{H}^{T} P+P A_{H}+K^{T} B_{H}^{T} P+P B_{H} K\right) x .
$$

The product of a matrix and its transpose is a positive semidefinite matrix. Then

$$
\begin{gathered}
\left(P A_{H}-I\right)\left(A_{H}^{T} P-I\right)=P A_{H} A_{H}^{T} P+I-A_{H}^{T} P-P A_{H} \geq 0, \\
\left(K^{T}-P B_{H}\right)\left(K-B_{H}^{T} P\right)=K^{T} K+P B_{H} B_{H}^{T} P-K^{T} B_{H}^{T} P-P B_{H} K \geq 0 .
\end{gathered}
$$

Substituting (13) to (12), yields the following upper constraint for $\dot{\mathcal{V}}(x)$

$$
\dot{\mathcal{V}}(x) \leq x^{T}\left(A_{\mathrm{cl}}^{T} P+P A_{\mathrm{cl}}+P A_{H} A_{H}^{T} P+I+K^{T} K+P B_{H} B_{H}^{T} P\right) x \leq 0 .
$$

Forcing the above upper bound of $\dot{\mathcal{V}}(x)$ to be negative semidefinite, a sufficient condition for stability of the system (9) is achieved. Using the Schur complement [26], the inequality (14) is equivalent to the following matrix inequality

$$
\left[\begin{array}{ccccc}
A_{\mathrm{cl}}^{T} P+P A_{\mathrm{cl}} & P A_{H} & P B_{H} & I & K^{T} \\
A_{H}^{T} P & -I & 0 & 0 & 0 \\
B_{H}^{T} P & 0 & -I & 0 & 0 \\
I & 0 & 0 & -I & 0 \\
K & 0 & 0 & 0 & -I
\end{array}\right] \leq 0
$$

The non-strictness of the matrix inequality above stems from the zero eigenvalues of matrix $A$. The matrix inequality (15) cannot be solved directly by the available LMI tools because of the product 
of variables in $\left(A_{\mathrm{cl}}^{T} P+P A_{\mathrm{cl}}\right)$. However, by taking the standard change of variables as in (10), the inequality (15) can be turned into an LMI, which can be solved by LMI tools [26]. From pre- and post-multiplying (15) by a symmetric congruence transformation $\operatorname{diag}(\mathbf{X}, I, I, I, I)$, the LMI (11) with variables $\mathbf{X}>0, \mathbf{Y}$ derives. Note that the standard variable transformation (10) does not introduce conservatism [26].

The structure constraints of the variables $\mathbf{X}$ and $\mathbf{Y}$ in (10) are introduced in order to derive a block-diagonal controller $K$ for the overall system. Each inverter is then stabilized by a decentralized controller $K_{i}$. This note also completes the proof.

Remark 3: It is clear that the decentralized control approach is generally more conservative than the centralized control, where no structure constraint on the LMI variables is needed. The feasibility of the LMI (11) depends on the inevitable interconnection between inverters, whereas the LMI achieves the highest feasibility chance in the case of no or a slight interconnection. In addition, the conservatism of the theorem 1 arises also from the inequality (14), where instead of forcing $\dot{\mathcal{V}}(x)$ to be negative semidefinite, the negative semidefiniteness of its upper bound is considered.

\section{QUASI-BLOCK DIAGONAL DOMINANCE}

As stated above, the inverters interact with each other via their control inputs and state variables, which are characterized by the off-diagonal parts $A_{H}$ and $B_{H}$ of the system matrices $A$ and $B$. The interconnection arises from the power exchanges between system nodes. More power exchanged implies a better power sharing performance. However, power sharing must be conducted within the stability margin of microgrids. From the control point of view, the interconnection can be seen as a disturbance on the inverters, and has a potential to alter the stability of the overall system. Therefore, there is a trade-off relation between stability and power sharing. A more robust system results in a worse power sharing performance.

From the stability perspective, it is desirable to decouple the inverters, i.e. restrict the power exchanges between inverters. Besides, as decentralized control for inverters is required, a less-coupled microgrid will allow local controllers to perform better control on inverters. Later, in a less-coupled microgrid, the influence of each inverter on the stability of the overall system is reduced. It is relevant to the plug-and-play structure of microgrids. That is, if some generation units are plugged in or lost during operation, the system stability is not significantly affected.

Hence, in this section it will show that by solving a modified version of the LMI (11) with an additional $0<\gamma \ll 1$ in the diagonal elements, the resulting controller will increase the blockdiagonal dominant degree of the closed-loop system (9), which in turn decreases the influence of the interconnection between inverters on the system stability. The block-diagonal dominance of a transfer matrix is defined in the following.

Definition 1: [27] Let us consider an $m \times m$ complex matrix $\mathcal{Q}$, which is decomposed as $\mathcal{Q}=$ $\mathcal{Q}_{d}+\mathcal{Q}_{H}$, where

$$
\mathcal{Q}_{d}=\operatorname{diag}\left(\mathcal{Q}_{1}, \ldots, \mathcal{Q}_{n}\right), \quad \mathcal{Q}_{H}=\left[\begin{array}{cccc}
\mathcal{Q}_{11} & \mathcal{Q}_{12} & \ldots & \mathcal{Q}_{1 n} \\
\mathcal{Q}_{21} & \mathcal{Q}_{22} & \ldots & \mathcal{Q}_{n n} \\
\vdots & \vdots & \ddots & \vdots \\
\mathcal{Q}_{n 1} & \mathcal{Q}_{n 2} & \ldots & \mathcal{Q}_{n n}
\end{array}\right]
$$

$\mathcal{Q}_{i}$ and $\mathcal{Q}_{i j}$, for $i=1, \ldots, n$, are, respectively, $m_{i} \times m_{i}$ and $m_{i} \times m_{j}$ submatrices of $\mathcal{Q}_{d}$ and $\mathcal{Q}_{H}$, and $m=m_{1}+\cdots+m_{n}$. Note that the diagonal blocks of $\mathcal{Q}$ are $\mathcal{Q}_{i}+\mathcal{Q}_{i i}$, where $\mathcal{Q}_{i i}$ may be zero 
or nonzero matrix and is introduced to reflect any uncertainty in the diagonal blocks of $\mathcal{Q}$. Then, a matrix $\mathcal{Q}$ is said to be quasi-block diagonal dominant if there exists a matrix norm $\|\cdot\|$ such is

$$
\left\|\mathcal{Q}_{H} \mathcal{Q}_{d}^{-1}\right\|<1 .
$$

In order to investigate the quasi-block diagonal dominance of the closed-loop system (9), the Laplace transform of the open-loop system (6) with a reference input $R(s)$ is taken as follows

$$
\left\{\begin{aligned}
s X(s) & =A_{d} X(s)+B_{d} U(s)+A_{H} X(s)+B_{H} U(s) \\
U(s) & =K(X(s)-R(s)) \\
Y(s) & =X(s)
\end{aligned}\right.
$$

An equivalent representation of the closed-loop system (9) in the Laplace transform is

$$
\left\{\begin{aligned}
s \Pi \tilde{X}(s) & =\tilde{A} \tilde{X}(s)-\tilde{B} K R(s)+\tilde{H} \tilde{X}(s), \\
Y(s) & =\tilde{C} \tilde{X}(s),
\end{aligned}\right.
$$

where $\Pi \in \mathbb{R}^{5 n \times 5 n}, \tilde{X} \in \mathbb{R}^{5 n \times 1}, \tilde{A} \in \mathbb{R}^{5 n \times 5 n}, \tilde{B} \in \mathbb{R}^{5 n \times 2 n}, \tilde{H} \in \mathbb{R}^{5 n \times 5 n}, \tilde{C} \in \mathbb{R}^{3 n \times 5 n}$ as

$$
\Pi=\left[\begin{array}{ll}
I & 0 \\
0 & 0
\end{array}\right], \tilde{X}=\left[\begin{array}{l}
X(s) \\
U(s)
\end{array}\right], \tilde{A}=\left[\begin{array}{cc}
A_{d} & B_{d} \\
K & -I
\end{array}\right], \tilde{B}=\left[\begin{array}{l}
0 \\
I
\end{array}\right], \tilde{H}=\left[\begin{array}{cc}
A_{H} & B_{H} \\
0 & 0
\end{array}\right], \tilde{C}=\left[\begin{array}{ll}
I & 0
\end{array}\right] .
$$

It is noteworthy that the descriptor system representation above is explicitly used to achieve a sufficient condition for the quasi-block diagonal dominance of the closed-loop system. The controller design is executed for the usual system representation (9). From $L=(s \Pi-\tilde{A})^{-1}$ then, the transfer function matrix of the closed-loop system (19) from $R(s)$ to $Y(s)$ derives as follows

$$
\begin{aligned}
\tilde{G}_{\mathrm{cl}}(s) & =-\tilde{C}(s \Pi-\tilde{A}-\tilde{H})^{-1} \tilde{B} K=-\tilde{C}\left(I-(s \Pi-\tilde{A})^{-1} \tilde{H}\right)^{-1}(s \Pi-\tilde{A})^{-1} \tilde{B} K \\
& =-\tilde{C}(I-L \tilde{H})^{-1} L \tilde{B} K .
\end{aligned}
$$

If $\|L \tilde{H}\|_{\infty}<\gamma \ll 1$, then $(I-L \tilde{H})^{-1} \cong I+L \tilde{H}[21]$. The following is then derived

$$
\tilde{G}_{\mathrm{cl}}(s) \cong-\tilde{C}(I+L \tilde{H}) L \tilde{B} K=-\tilde{C} L \tilde{H} L \tilde{B} K-\tilde{C} L \tilde{B} K
$$

It will show that $\|L \tilde{H}\|_{\infty}<\gamma \ll 1$ is also the condition to increase the quasi-block diagonal dominance of the closed-loop system. Moreover, $\tilde{C} L \tilde{B} K$ is the transfer function of the closed-loop block-diagonal part of the overall system as

$$
\tilde{C} L \tilde{B} K=\tilde{C}(s \Pi-\tilde{A})^{-1} \tilde{B} K=\left(s I-A_{d}-B_{d} K\right)^{-1} B_{d} K .
$$

Then, $\tilde{C} L \tilde{H} L \tilde{B} K$ is the off-diagonal part of the closed-loop system $\tilde{G}_{\mathrm{cl}}(s)$ resulting in

$$
\begin{aligned}
\tilde{C} L \tilde{H} L \tilde{B} K & =\left(s I-A_{d}-B_{d} K\right)^{-1} A_{H}\left(s I-A_{d}-B_{d} K\right)^{-1} B_{d} K \\
& +\left(s I-A_{d}-B_{d} K\right)^{-1} B_{H} K\left(I+\left(s I-A_{d}-B_{d} K\right)^{-1} B_{d} K\right) .
\end{aligned}
$$

$B_{H}$ contains off-diagonal matrices $B_{i j}, i \neq j, i, j=1, \ldots, n$, given in (7). It is seen that $B_{H}$ characterizes the interconnection between inverters through voltages. Stemming from the local behavior of inverter output voltages, $B_{H}$ is in fact relatively small compared with $B_{d}$. Furthermore, it will be shown that the controller $K$ with smaller gains results in a more quasi-block diagonal 
dominant closed-loop system. Accordingly, the product of a block diagonal matrix $K$ with an offdiagonal matrix $B_{H}$ results in a relatively small $B_{H} K$, which makes the second right hand term of the expression above relatively small compared to the first term. Thus, the following approximation of (23) is adopted (see [28] for more mathematical details of this approximation) $)^{3}$

$$
\tilde{C} L \tilde{H} L \tilde{B} K \cong\left(s I-A_{d}-B_{d} K\right)^{-1} A_{H}\left(s I-A_{d}-B_{d} K\right)^{-1} B_{d} K=\tilde{C} L \tilde{H} \tilde{C}^{+} \tilde{C} L \tilde{B} K,
$$

where $\tilde{C}^{+}=\left[\begin{array}{ll}I & 0\end{array}\right]^{T}$ is the left pseudo-inverse of $\tilde{C}$.

By considering $\mathcal{Q}_{d}=\tilde{C} L \tilde{B} K$ and $\mathcal{Q}_{H}=\tilde{C} L \tilde{H} L \tilde{B} K$, yields the following $\mathcal{H}_{\infty}$ norm

$$
\begin{aligned}
\left\|\mathcal{Q}_{H} \mathcal{Q}_{d}^{-1}\right\|_{\infty} & =\left\|\tilde{C} L \tilde{H} L \tilde{B} K(\tilde{C} L \tilde{B} K)^{-1}\right\|_{\infty} \cong\left\|\tilde{C} L \tilde{H} \tilde{C}^{+} \tilde{C} L \tilde{B} K(\tilde{C} L \tilde{B} K)^{-1}\right\|_{\infty} \\
& \leq\|\tilde{C} L \tilde{H}\|_{\infty} \underbrace{\left\|\tilde{C}^{+}\right\|_{\infty}}_{=1}\|\underbrace{\tilde{C} L \tilde{B} K(\tilde{C} L \tilde{B} K)^{-1}}_{=I}\|_{\infty}=\|\tilde{C} L \tilde{H}\|_{\infty} .
\end{aligned}
$$

Then, a sufficient condition for the closed-loop system (9) to be quasi-block diagonal dominant with respect to the definition 1 is obtained as follows

$$
\left\|\mathcal{Q}_{H} \mathcal{Q}_{d}^{-1}\right\|_{\infty} \leq\|\tilde{C} L \tilde{H}\|_{\infty} \leq\|\tilde{C}\|_{\infty}\|L \tilde{H}\|_{\infty}=\|L \tilde{H}\|_{\infty}<\gamma \ll 1 .
$$

Moreover, $\|L \tilde{H}\|_{\infty}$ is originally given by

$$
\begin{aligned}
\|L \tilde{H}\|_{\infty} & =\left\|\left[\begin{array}{cc}
s I-A_{d} & -B_{d} \\
-K & I
\end{array}\right]^{-1}\left[\begin{array}{cc}
A_{H} & B_{H} \\
0 & 0
\end{array}\right]\right\|_{\infty} \\
& =\left\|\left[\begin{array}{c}
\left(s I-A_{d}-B_{d} K\right)^{-1} \\
K\left(s I-A_{d}-B_{d} K\right)^{-1}
\end{array}\right]\left[A_{H} B_{H}\right]\right\|_{\infty}=\left\|\hat{C}\left(s I-A_{\mathrm{cl}}\right)^{-1} \hat{B}\right\|_{\infty},
\end{aligned}
$$

where $\hat{B}=\left[\begin{array}{ll}A_{H} & B_{H}\end{array}\right]$ and $\hat{C}=\left[\begin{array}{ll}I & K^{T}\end{array}\right]^{T}$. Hence, $\|L \tilde{H}\|_{\infty}$ is equal to the $\mathcal{H}_{\infty}$ norm of the transfer function matrix of the system $\left(A_{\mathrm{cl}}, \hat{B}, \hat{C}, 0\right)$.

By applying the Bounded Real Lemma [26] to the system $\left(A_{\mathrm{cl}}, \hat{B}, \hat{C}, 0\right)$, the matrix $A_{\mathrm{cl}}$ is stable and the $\mathcal{H}_{\infty}$ norm of its transfer function matrix is less than $\gamma \ll 1$, which is equivalent to $\|L \tilde{H}\|_{\infty}<\gamma \ll 1$, if and only if there exists a positive definite matrix $P$, so that the following matrix inequality is feasible

$$
\left[\begin{array}{ccccc}
A_{\mathrm{cl}}^{T} P+P A_{\mathrm{cl}} & P A_{H} & P B_{H} & I & K^{T} \\
A_{H}^{T} P & -\gamma I & 0 & 0 & 0 \\
B_{H}^{T} P & 0 & -\gamma I & 0 & 0 \\
I & 0 & 0 & -\gamma I & 0 \\
K & 0 & 0 & 0 & -\gamma I
\end{array}\right]<0
$$

Remark 4: With $\gamma \in(0,1]$, the matrix inequality (28) serves as a sufficient condition for the matrix inequality (15). Hence, a solution of (28) also guarantees the stability of the closed-loop system (9).

By using the variable transformation (10), the inequality (28) is turned into the following modified version of the LMI (11) with an additional $\gamma$ in the diagonal elements

$$
\left[\begin{array}{ccccc}
A_{d} \mathbf{X}+B_{d} \mathbf{Y}+\left(A_{d} \mathbf{X}+B_{d} \mathbf{Y}\right)^{T} & A_{H} & B_{H} & \mathbf{X} & \mathbf{Y}^{T} \\
A_{H}^{T} & -\gamma I & 0 & 0 & 0 \\
B_{H}^{T} & 0 & -\gamma I & 0 & 0 \\
\mathbf{X} & 0 & 0 & -\gamma I & 0 \\
\mathbf{Y} & 0 & 0 & 0 & -\gamma I
\end{array}\right]<0 .
$$

\footnotetext{
${ }^{3}$ The simulation in section 5 confirms the accuracy of the assumption (24).
} 
By solving the LMI (29) with a small enough $\gamma>0$, the derived controller guarantees the stability of the system (9) and its quasi-block diagonal dominant degree of $\gamma$.

Remark 5: It is now clear that the descriptor system form (19) is introduced in order to formulate the convex LMI optimization problem (29), whose feasible results satisfy two control targets: stability and quasi-block diagonal dominance. Although the transfer function matrix of the closed-loop system can be achieved from (18) as $G_{\mathrm{cl}}(s)=-\left(s I-A_{d}-B_{d} K-A_{H}-B_{H} K\right)^{-1} B K$, the matrix $B$ is a full matrix, which makes the formulation of a convex LMI problem not trivial and straightforward to achieve as in the case of $\tilde{G}_{\mathrm{cl}}(s)$. Even if $B_{H} K$ is neglected, the LMI condition for $G_{\mathrm{cl}}(s)$ to be quasi-block diagonal dominant is absolutely different from the LMI (11), which does not guarantee the stability of the closed-loop system.

\section{AN ACADEMICAL EXAMPLE}

In this section, an academical example is provided to show the effectiveness of the proposed control approach. Consider the system shown in Fig. 3, which consists of two inverters and loads.

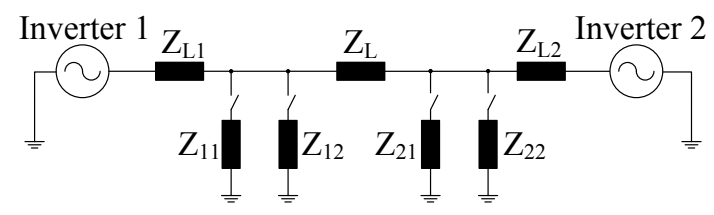

Figure 3: Test system with two inverters and loads.

Parameters of the test system are provided in table 1. Simulation is conducted in Simulink/Matlab. A sequence of discrete events separated by five seconds intervals is included in the simulation according to the following scenario: initially, $Z_{11}$ and $Z_{21}$ are connected to the system, then $Z_{12}$ and $Z_{22}$ are successively added in the written order; finally the reference changes (by applying $\tilde{P}_{i_{0}}:=\tilde{P}_{i_{0}}+\Delta P_{i}, \tilde{Q}_{i_{0}}:=\tilde{Q}_{i_{0}}+\Delta Q_{i}$ to the control loop in Fig. 2) are included into the simulation. The equilibrium active and reactive power are calculated by solving the power flow equations (1), using the admittance matrix $Y$ of the system at nominal operating frequency.

In the controller design procedure, the system variables are converted into per unit values for computational simplicity by eliminating units and expressing system quantities as dimensionless ratios [29]. The LMI problems are solved by using Yalmip toolbox [30] and SeDuMi solver [31] in Matlab. From solving the LMI problem (29), the following controllers with their actual values are derived

$$
\begin{aligned}
& K_{1}=\left[\begin{array}{ccc}
-1.824 & 0.0003 & -0.0002 \\
2 \cdot 10^{-5} & -0.127 & -0.0134
\end{array}\right], K_{1}=\left[\begin{array}{ccc}
-1.824\left[\frac{1}{s}\right] & 2.4 \cdot 10^{-6}\left[\frac{\mathrm{rad}}{\mathrm{skW}}\right] & -2 \cdot 10^{-6}\left[\frac{\mathrm{rad}}{\mathrm{skVar}}\right] \\
0.02\left[\frac{\mathrm{V}}{\mathrm{rad}}\right] & -1.2\left[\frac{\mathrm{V}}{\mathrm{kW}}\right] & -0.13\left[\frac{\mathrm{V}}{\mathrm{kVar}}\right]
\end{array}\right], \\
& K_{2}=\left[\begin{array}{lrr}
-1.824 & -0.001 & 0.0003 \\
-0.0002 & -0.1002 & -0.057
\end{array}\right], K_{2}=\left[\begin{array}{ccc}
-1.824\left[\frac{1}{s}\right] & -8 \cdot 10^{-6}\left[\frac{\mathrm{rad}}{\mathrm{skW}}\right] & 3 \cdot 10^{-6}\left[\frac{\mathrm{rad}}{\mathrm{skVar}}\right] \\
-0.251\left[\frac{\mathrm{V}}{\mathrm{rad}}\right] & -1\left[\frac{\mathrm{V}}{\mathrm{kW}}\right] & -0.55\left[\frac{\mathrm{V}}{\mathrm{kVar}}\right]
\end{array}\right] \text {. }
\end{aligned}
$$

The controllers $K_{1}$ and $K_{2}$ return a highly quasi-block diagonal dominant degree ${ }^{4}$ of the closedloop system, $\gamma=0.0028$. By solving the LMI (29) with a larger $\gamma$, the controller gains become larger, e.g., with $\gamma=0.7,|\max (K[i, j])|=-9.54$.

\footnotetext{
${ }^{4}$ The accuracy of the approximation in $(24)$ is numerically verified as $\|\tilde{C} L \tilde{H} L \tilde{B} K\|_{\infty} \cong$ $\left\|\tilde{C} L \tilde{H} \tilde{C}^{+} \tilde{C} L \tilde{B} K\right\|_{\infty}=0.0015$. The quasi-block diagonal dominance is $\left\|Q_{H} Q_{d}^{-1}\right\|_{\infty}=0.19$.
} 
Table 1: Test system parameters

\begin{tabular}{|c|c|c|}
\hline Voltages and & $V_{1_{0}}=1029[\mathrm{~V}]$ & $\delta_{1_{0}}=0.000[\mathrm{rad}]$ \\
phase angles & $V_{2_{0}}=1030+1.3 i[\mathrm{~V}]$ & $\delta_{2_{0}}=0.0013[\mathrm{rad}]$ \\
\hline Active and & $\tilde{P}_{1_{0}}=9.75[\mathrm{~kW}]$ & $\tilde{P}_{2_{0}}=16.72[\mathrm{~kW}]$ \\
reactive power & $\tilde{Q}_{1_{0}}=4.048[\mathrm{kVar}]$ & $\tilde{Q}_{2_{0}}=7.33[\mathrm{kVar}]$ \\
\hline Base values & $S_{\text {base }}=108[\mathrm{kVA}]$ & $V_{\text {base }}=1030[\mathrm{~V}]$ \\
& $Z_{\text {base }}=9.823[\Omega]$ & $\delta_{\text {base }}=1[\mathrm{rad}]$ \\
\hline Load & $Z_{11}=108+i 10.8[\Omega]$ & $Z_{21}=45+i 28[\Omega]$ \\
impedances & $Z_{12}=69+i 15[\Omega]$ & $Z_{22}=60+i 19[\Omega]$ \\
\hline Line impedance & $Z_{\mathrm{L}_{1}}=0.4+i 0.4[\Omega]$ & $Z_{\mathrm{L}}=0.8+i 0.42[\Omega]$ \\
& $Z_{\mathrm{L}_{2}}=0.4+i 0.3[\Omega]$ & \\
\hline Nominal frequency & $\omega_{\text {nom }}=2 \pi 50[\mathrm{rad} / \mathrm{s}]$ & \\
\hline Filter parameters & $\tau_{1}=0.0265[\mathrm{~s}]$ & $\tau_{2}=0.0265[\mathrm{~s}]$ \\
\hline Reference changes & $\Delta P_{1}=10[\mathrm{~kW}]$ & $\Delta Q_{1}=5[\mathrm{kVar}]$ \\
& $\Delta P_{2}=5[\mathrm{~kW}]$ & $\Delta Q_{2}=8[\mathrm{kVar}]$ \\
\hline
\end{tabular}

Remark 6: It is seen from (30) that the actual values of the controller gains associated to the active and reactive power are relatively small. However, it is normal, if those actual controller gains are compared to the typical droop gains in $[3,5,22]$.

The simulation results are displayed in Fig. 4. The transfer processes in Fig. 4 show that the system is stabilized robustly after perturbations by the controllers. As stated earlier, one of the benefits of our proposed control scheme is that frequency deviations always converge towards zero, whereas voltages and phase angles vary around their nominal values to adjust the output power. It can be seen that after perturbations the new equilibrium points differ from the (initial) nominal equilibrium point. When a load is connected, it is supplied by both inverters. Thus, there is a sort of power sharing, though the power portions injected by the inverters are not the same, even when the controller gains of both inverters are much alike.

Concerning the voltage performance, variations of voltage magnitudes are acceptable. However, magnitude variations of voltages can be large if load changes are large. The purpose of the power reference changes at $t=30[s]$ and $t=40[s]$ is to reduce the power mismatches between the generated power and the references. It is seen that the deviations of $V_{1}$ and $V_{2}$ are reduced.

\section{CONCLUSION}

The system stability is defined as the most important control target of inverter-based microgrids. A power control loop for inverters and a LMI condition to guarantee the stability of an overall microgrid and zero steady-state frequency deviations based only on local measurements are proposed. Then, the LMI optimization problem is extended to decouple the closed-loop microgrid. This results in some advantages, such as the improvement of the stability margin and the decreasing influence of the interconnection on the system stability. The system stability and the performance of frequencies and voltages with respect to small load changes are guaranteed as illustrated by a simulation.

Although there are power exchanges between system nodes, the power sharing performance is not guaranteed. In the next step of our research, the authors will consider the apparent disadvantage of decoupled microgrids, namely, the low power sharing performance. Although the voltage perfor- 

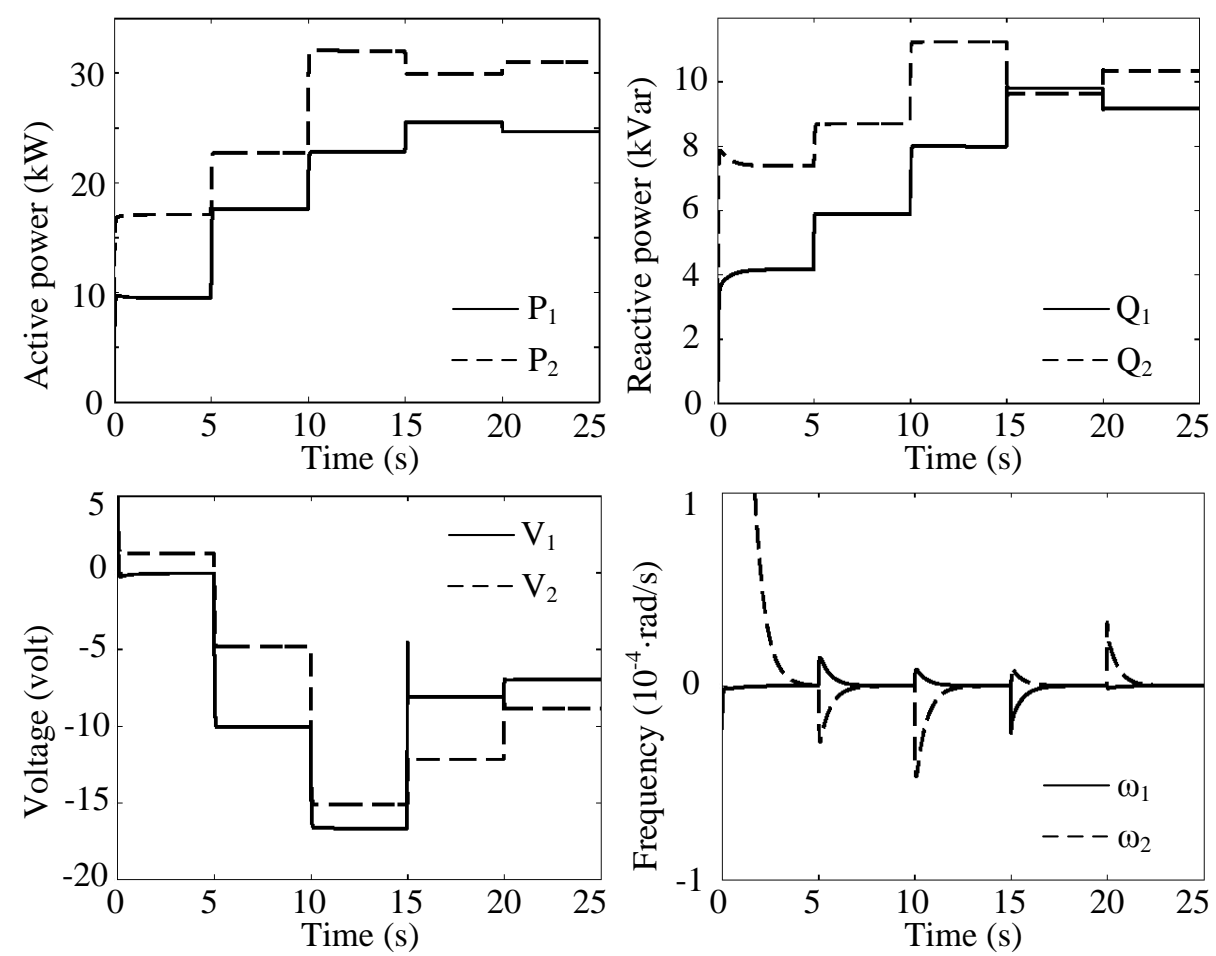

Figure 4: Transfer processes of the test system.

mance is guaranteed with respect to small load changes, it is expected that with larger load changes, magnitude variations of voltages will also become larger. This can cause functionality problems to connected loads of a microgrid. Thus, based on the work done so far, the authors will investigate a condition to robustly guarantee not only the system stability but also the voltage performance of inverter-based microgrids.

\section{REFERENCES}

[1] R. Lasseter and P. Paigi, "Microgrid: a conceptual solution," in Power Electronics Specialists Conference, 2004. PESC 04. 2004 IEEE 35th Annual, vol. 6, June 2004, pp. 4285-4290.

[2] N. Hatziargyriou, H. Asano, R. Iravani, and C. Marnay, "Microgrids," Power and Energy Magazine, IEEE, vol. 5, no. 4, pp. 78-94, July 2007.

[3] N. Pogaku, M. Prodanovic, and T. Green, "Modeling, analysis and testing of autonomous operation of an inverter-based microgrid," Power Electronics, IEEE Transactions on, vol. 22, no. 2, pp. 613-625, March 2007.

[4] K. De Brabandere, B. Bolsens, J. Van den Keybus, A. Woyte, J. Driesen, and R. Belmans, "A voltage and frequency droop control method for parallel inverters," Power Electronics, IEEE Transactions on, vol. 22, no. 4, pp. 1107-1115, July 2007.

[5] M. Chandorkar, D. Divan, and R. Adapa, "Control of parallel connected inverters in standalone ac supply systems," Industry Applications, IEEE Transactions on, vol. 29, no. 1, pp. 136-143, Jan. 1993. 
[6] T. D. Trung, "A robust decentralized controller design for inverter-based microgrids with dynamic loads," in Industrial Electronics Society, IECON 2013 - 39th Annual Conference of the IEEE, Nov. 2013, pp. 1507-1512.

[7] J. Schiffer, A. Anta, T. D. Trung, J. Raisch, and T. Sezi, "On power sharing and stability in autonomous inverter-based microgrids," in Decision and Control (CDC), 2012 IEEE 51st Annual Conference on, Dec. 2012, pp. 1105-1110.

[8] J. Guerrero, J. Matas, L. de Vicua, M. Castilla, and J. Miret, "Wireless-control strategy for parallel operation of distributed-generation inverters," Industrial Electronics, IEEE Transactions on, vol. 53, no. 5, pp. 1461-1470, Oct. 2006.

[9] A. Roslan, K. Ahmed, S. Finney, and B. Williams, "Improved instantaneous average currentsharing control scheme for parallel-connected inverter considering line impedance impact in microgrid networks," Power Electronics, IEEE Transactions on, vol. 26, no. 3, pp. 702-716, March 2011.

[10] T. Vandoorn, B. Meersman, J. De Kooning, and L. Vandevelde, "Controllable harmonic current sharing in islanded microgrids: Dg units with programmable resistive behavior toward harmonics," Power Delivery, IEEE Transactions on, vol. 27, no. 2, pp. 831-841, April 2012.

[11] J. Guerrero, J. Vasquez, J. Matas, L. de Vicua, and M. Castilla, "Hierarchical control of droopcontrolled ac and dc microgrids - a general approach toward standardization," Industrial Electronics, IEEE Transactions on, vol. 58, no. 1, pp. 158-172, Jan. 2011.

[12] C.-T. Lee, C.-C. Chu, and P.-T. Cheng, "A new droop control method for the autonomous operation of distributed energy resource interface converters," in Energy Conversion Congress and Exposition (ECCE), 2010 IEEE, Sept. 2010, pp. 702-709.

[13] J. Guerrero, J. Matas, L. G. de Vicuna, M. Castilla, and J. Miret, "Decentralized control for parallel operation of distributed generation inverters using resistive output impedance," Industrial Electronics, IEEE Transactions on, vol. 54, no. 2, pp. 994-1004, April 2007.

[14] H.-P. Beck and R. Hesse, "Virtual synchronous machine," in Electrical Power Quality and Utilisation, 2007. EPQU 2007. 9th International Conference on, Oct. 2007, pp. 1-6.

[15] R. Hesse, D. Turschner, and H. Beck, "Microgrid stabilization using the virtual synchronous machine (visma)," International Conference on Renewable energies and power quality, pp. 472 475, April 2009.

[16] Q.-C. Zhong and G. Weiss, "Synchronverters: Inverters that mimic synchronous generators," Industrial Electronics, IEEE Transactions on, vol. 58, no. 4, pp. 1259-1267, April 2011.

[17] G. Azevedo, F. Bradaschia, M. Cavalcanti, F. Neves, J. Rocabert, and P. Rodriguez, "Safe transient operation of microgrids based on master-slave configuration," in Energy Conversion Congress and Exposition (ECCE), 2011 IEEE, Sept. 2011, pp. 2191-2195.

[18] M. Prodanovic and T. Green, "High-quality power generation through distributed control of a power park microgrid," Industrial Electronics, IEEE Transactions on, vol. 53, no. 5, pp. 14711482, Oct. 2006.

[19] F. Dorfler and F. Bullo, "Kron reduction of graphs with applications to electrical networks," Circuits and Systems I: Regular Papers, IEEE Transactions on, vol. 60, no. 1, pp. 150-163, Jan. 2013. 
[20] R. Majumder, "Some aspects of stability in microgrids," Power Systems, IEEE Transactions on, vol. 28, no. 3, pp. 3243-3252, Aug. 2013.

[21] K. Zhou and J. Doyle, Essentials of Robust Control. Prentice Hall, 1998.

[22] R. Majumder, B. Chaudhuri, A. Ghosh, R. Majumder, G. Ledwich, and F. Zare, "Improvement of stability and load sharing in an autonomous microgrid using supplementary droop control loop," Power Systems, IEEE Transactions on, vol. 25, no. 2, pp. 796-808, May 2010.

[23] A. Phadke, "Synchronized phasor measurements in power systems," Computer Applications in Power, IEEE, vol. 6, no. 2, pp. 10-15, April 1993.

[24] J. De La Ree, V. Centeno, J. Thorp, and A. Phadke, "Synchronized phasor measurement applications in power systems," Smart Grid, IEEE Transactions on, vol. 1, no. 1, pp. 20-27, June 2010.

[25] B. Labibi, H. Marquez, and T. Chen, "Lmi optimization approach to robust decentralized controller design," International Journal of Robust and Nonlinear Control, vol. 21, no. 8, pp. 904924, 2011.

[26] C. Scherer and S. Weiland, Linear matrix inequalities in control, Lecture Notes. Dutch Institute for Systems and Control, Delft, The Netherlands, 2000.

[27] Y. Ohta, D. Siljak, and T. Matsumoto, "Decentralized control using quasi-block diagonal dominance of transfer function matrices," Automatic Control, IEEE Transactions on, vol. 31, no. 5, pp. 420-430, May 1986.

[28] B. Labibi, B. Lohmann, A. Khaki Sedigh, and P. Jabedar Maralani, "Output feedback decentralized control of large-scale systems using weighted sensitivity functions minimization," Systems E control letters, vol. 47, no. 3, pp. 191-198, 2002.

[29] P. Kundur, Power system stability and control. McGraw-Hill, 1994.

[30] J. Lofberg, "Yalmip : a toolbox for modeling and optimization in matlab," in Computer Aided Control Systems Design, 2004 IEEE International Symposium on, Sept. 2004, pp. 284-289.

[31] J. F. Sturm, "Using sedumi 1.02, a matlab toolbox for optimization over symmetric cones," Optimization methods and software, vol. 11, no. 1-4, pp. 625-653, 1999.

Received on December 13 - 2014

Revised on February 27 - 2015 